\title{
Special Issue: Information Dynamics in Artificial Societies (IDAS@ESSLLI-14)
}

\author{
Emiliano Lorini $^{1}$ - Laurent Perrussel ${ }^{1}$. \\ Roland Mühlenbernd ${ }^{2}$
}

Published online: 7 October 2016

(C) Springer Science+Business Media Dordrecht 2016

This volume includes extended versions of a selection of the best papers presented at the workshop Information Dynamics in Artificial Societies (IDAS) and its special track on Sociolinguistics and Network Games (SLANG), of the 26th European Summer School in Logic, Language and Information (ESSLLI), 2014. This workshop focused on information exchange between several artificial agents. More precisely, the workshop aimed at discussing formal theories showing:

- how information circulates in an artificial society by direct interaction, communication or signaling;

- how it influences the individual cognitive attitudes (e.g. beliefs) and the collective attitudes (e.g. judgment);

- how behavioral characteristics of information exchange (e.g. signaling conventions) emerge in repeated interactions.

The papers presented at IDAS and SLANG gave a broad and diverse overview of ongoing research on these topics: the program included ongoing research about concurrent information change, normative change, social influence, merging, aggregation, signaling strategies and cellular games. The workshop chairs selected the best papers presented in these workshops. Authors were asked to submit extended and updated versions of their papers. These went through a second round of a thorough review process, resulting in the five articles included in this special issue.

The work presented in this special issue includes developments on norm dynamics, social influence, judgment aggregation and signaling strategies. These developments stress the multidisciplinary dimension of the field: the authors are researchers from

\footnotetext{
$凶 \quad$ Laurent Perrussel

laurent.perrussel@ut-capitole.fr

1 IRIT -Université de Toulouse, Toulouse, France

2 Eberhard Karls University Tübingen, Tübingen, Germany
} 
game theory, linguistics and knowledge representation domains. An overview of the five papers in this special section follows.

The first paper is about normative dynamics. Norms constraint the dynamics of an artificial society and studying the dynamics of a set of rules is at first importance. The dynamics of individual epistemic states has been addressed by Alchourrón, Gärdenfors and Makinson (AGM) with their seminal work about belief revision. However, how a set of rules may change is still an open question. Boella, Pigozzi and van der Torre address this research problem in their paper AGM Contraction and Revison of Rules. They study whether the AGM framework is suitable for describing the dynamics of a set of rules. The authors show that, once rules are encoded in input/output logic, an AGM like framework can be defined for describing classical change operation: expansion, contraction and revision. A second important result is the new version of partial meet contraction suitable for operating on a set of rules.

The second paper focuses on social influence. If we assume an influence relation between agents, assessing the impact of this relation consists of assessing how an agent changes its belief state in order to adopt the opinion of its influencers. In their paper Reflecting on social influence in networks, Hansen, Christoff and Proietti propose a logical system for formally representing social influence. The proposed logic enables to distinguish public and private opinions and to characterize social influence through (1) information propagation, and (2) interplay rules between public and private opinions. These rules represent a significant and interesting contribution as they clearly show how opinion changes when agents face agreement or disagreement between influencers.

The third paper is about judgment aggregation which is an important aspect of artificial societies in which collective decisions have to be made. Judgment aggregation is usually considered from the perspective of algorithmic decision theory, i.e. the operational semantics. In his paper Natural Deduction for Modal Logic of Judgment Aggregation, Perkov provides a different perspective: a modal logic with inference rules describing aggregation. The proposed system is sound and complete. The author shows not only how this modal logic is helpful for deriving properties about some judgment aggregation procedures but, also how inference rules of the logic support reasoning about agenda and profiles in a natural way.

The fourth and fifth paper focus on the puzzle of the emergence of compositionality, which appears to be a central feature in natural languages. The authors of these two papers use (1) the signaling game (Lewis 1969), a game-theoretic model that models communication between two agents, a sender and a receiver, and (2) the learning rule reinforcement learning (Roth and Erev 1995) to show that compositionality can emerge in repeated communication of unsophisticated agents.

In his paper The Evolution of Compositionality in Signaling Games, Franke presents an explanation for how natural languages might have become compositional. He shows that composition-like signaling behavior can emerge without being engineered-in from the beginning by using a conservative generalization of the learning model, which he calls spill-over reinforcement learning.

In the fifth paper Compositional Signaling in a Complex World, Steinert-Threlkeld presents an explanation for why natural languages might have become compositional. His study reveals that compositional signaling is evolutionary beneficial in a complex 
world: virtual experiments show that in comparison to atomic signaling, compositional signaling leads to more effective communication iff there is a considerable number of different states of the world which are essential to be distinguishably communicated by the agents.

We appreciate the dedication and professional work of all the reviewers of this special issue, and the patience and guidance of Sylvain Salvati, JLLI Editor.

Emiliano, Laurent and Roland

\section{References}

Lewis, D. (1969). Convention. A philosophical study. Cambridge: Harvard University Press.

Roth, A. E., \& Erev, I. (1995). Learning in extensive form games: Experimental data and simple dynamical models in the intermediate term. Games and Economic Behavior, 8(1), 164-212. 